\title{
Scintillation Studies of the Pulsar PSR B0329+54
}

\author{
Daniel R. Stinebring and Michael D. Faison ${ }^{1}$ \\ Department of Physics, Oberlin College, Oberlin, OH 44074
}

Mark M. McKinnon

National Radio Astronomy Observatory, Green Bank, WV 24944

\begin{abstract}
Refractive and diffractive scintillation are thought to be two related effects of scattering in the interstellar medium. The predicted modulation of diffractive scintillation parameters by refractive effects has not been thoroughly tested, however. We report 24 days of scintillation observations of the radio pulsar PSR B0329+54 from September 1993 to June 1994 with a $26-\mathrm{m}$ telescope operating at $610 \mathrm{MHz}$. Dynamic spectra were obtained on each day, from which the characteristic bandwidth and time scale of the diffractive scintillation pattern were determined. A relative flux value for the pulsar was also obtained on each day. The cross-correlation coefficient for each unique pair of the observables flux, $\delta F$, bandwidth, $\delta \nu$ and time scale, $\delta t$ - was formed from these data. The observed values of these coefficients and their $67 \%$ confidenceintervals are: $C_{\delta F \delta \nu}=-0.4 \pm 0.2, C_{\delta F \delta t}=-0.7 \pm 0.1$, and $C_{\delta \nu \delta t}=$ $0.5 \pm 0.2$. Theoretical expectations are that $C_{\delta F \delta \nu}=-0.8, C_{\delta F \delta t}=$ -0.6 , and $C_{\delta \nu \delta t}=0.8$. We conclude that we have detected the expected modulation of diffractive parameters by refractive scintillation. This adds confidence to the interpretation that diffractive and refractive scintillation of compact radio sources are caused by the same underlying scattering process.
\end{abstract}

\section{Introduction}

Refraction by medium scale structures in the interstellar medium $\left(10^{9}--10^{12} \mathrm{~m}\right)$ is predicted to influence the more readily observable diffractive effects (Rickett 1990). A number of authors have addressed the effects of refraction on the dynamic spectra of pulsars, most recently Gupta, Rickett \& Lyne (1994); Cordes, Pidwerbetsky \& Lovelace (1986); and Romani, Narayan \& Blandford (1986). An anticorrelation between pulsar flux and decorrelation bandwidth is a central prediction of refractive scintillation theory and does not depend sensitively on the spectrum of inhomogeneities that gives rise to the scattering, as is clear from the explicit expressions for this and other correlation coefficients presented

${ }^{1}$ Dept. of Astronomy, University of Wisconsin, Madison, WI 53706 
by Romani, Narayan \& Blandford (1986). There is a similar prediction of an anticorrelation between pulsar flux and the characteristic time duration of scintillation maxima in a dynamic spectrum, the decorrelation time scale, $t_{d}$.

We present here an observational study of the correlation between pulsar flux - a measurement of refractive effects - and two diffractive observables: decorrelation bandwidth, $\nu_{d}$, and decorrelation time scale, $t_{d}$.

Our observations were made at a sky frequency of $610 \mathrm{MHz}$ with $20 \mathrm{MHz}$ of bandwidth and dual linearly-polarized feeds. The spectral processor was configured to produce a 256-channel spectrum in each polarization, giving a frequency resolution of $78 \mathrm{kHz}$. Spectra were binned into 128 time bins across the entire pulsar period, resulting in a time resolution of $5.6 \mathrm{~ms}$, which is approximately the full-width at half maximum of the pulse. Thus, we have little information about the variation of the spectrum across the pulse. Spectra in the two polarizations were collected once every 60 seconds and written to magnetic tape for off-line analysis. The duration of a single observation was about 90 minutes.

\section{Results and Conclusions}

Turning to our results, we see a statistically significant cross-correlation between all three pairings of observables. Furthermore, the sign of the cross-correlation coefficient is, in each case, consistent with that expected by theory: flux anticorrelates with both decorrelation bandwidth and time scale, and bandwidth and time scale are positively correlated with each other, with values given in the abstract. We consider this to be confirmation of the prediction that refractive scintillation modulates diffractive scintillation. Further details of this work are reported in Stinebring, Faison \& McKinnon (1996).

\section{References}

Cordes, J. M., Pidwerbetsky, A. \& Lovelace, R. V. E., 1986, ApJ, 310, 737

Gupta, Y., Rickett, B. J. \& Lyne, A. G., 1994, MNRAS, 269, 1035

Rickett, B. J., 1990, Ann Rev Astr Ap, 28, 561

Romani, R. W., Narayan, R. \& Blandford, R., 1986, MNRAS, 220, 19

Stinebring, D. R., Faison, M. D., \& McKinnon, M. M., 1996, ApJ, 460, 460 